\title{
Retropubic versus transobturator MUS: time to revisit?
}

\author{
Gunnar Lose $^{1}$ (D) $\cdot$ Niels Klarskov ${ }^{1}$
}

Received: 30 May 2017 / Accepted: 14 June 2017 / Published online: 4 July 2017

(C) The International Urogynecological Association 2017

Synthetic midurethral sling (MUS) operations have become the current standard for surgical treatment of stress urinary incontinence in women. The retropubic tension-free vaginal tape was introduced by Ulmsten et al. in 1996 [1], while the transobturator technique was introduced by Delorme in 2001 [2]. Thus, we have 20 and 15 years of accumulated evidence regarding these techniques, respectively. According to the latest Cochrane systematic review [3], the retropubic and transobturator procedures are associated with similar cure rates, both subjective and objective in the short term ( $\leq 1$ year), the medium term (1-5 years), and even in the long term ( $>5$ years). The relative risks vary between 1 and 0.95 .

The question, however, is whether these data provide a trustworthy description of efficacy in terms of durability of the MUS placed using the two procedures. A recent study based on a complete 10 -year national registry cohort of 8,671 women showed reoperation rates after 5 years for the retropubic and the transobturator approaches of $6 \%$ and $9 \%$, respectively. A Cox regression analysis revealed that the relative risk of reoperation after the transobturator procedure was twofold higher than after the retropubic procedure, a difference that was statistically significant [4]. The strength of these results is in the robustness of the information, since data reporting is compulsory for all hospitals according to Danish law and data completeness is above $90 \%$. Similarly, a recent report based on available National Health Service data in Scotland relating to 4,628 retropubic tape procedures

Gunnar Lose

Gunnar.Lose@regionh.dk

1 Department of Obstetrics and Gynecology, Herlev Gentofte Hospital, University of Copenhagen, Herlev Ringvej,

DK-2730 Herlev, Denmark and 4,850 transobturator tape procedures concluded that "beyond 12 months there is a substantially greater need for repeat SUI-surgery following the transobturator approach". Consequently, only the retropubic tape procedure is recommended for the surgical treatment of stress urinary incontinence [5].

Adding to the two national cohort studies the recent Cochrane review showed, that the relative reoperation rate after 1 year was 1.64 higher following the transobturator procedure, but the difference in the rates was not statistically significant. However, in the two medium-term studies and the four long-term studies the reoperation rates were 21.89 and 8.79 times higher, respectively, after the transobturator procedure, and the difference between these rates was significant [3]. All six studies pointed in the same direction. Finally, a recent systematic review and meta-analysis of retropubic versus transobturator MUS procedures in patients with "intrinsic sphincter deficiency" showed a lower subjective cure rate in the short/medium term and a higher reoperation rate in the long term after the transobturator procedure [6]. Thus, the transobturator approach may be especially inferior in patients with a weak closure mechanism (sphincter deficiency or poor support). Thus, accumulating evidence clearly indicates that the reoperation rate is significantly higher and hence slings placed are significantly less durable with the transobturator approach than with the retropubic approach.

Urethral slings work by creating midurethral support, and taking all the evidence together it is reasonable to state that slings placed using the retropubic approach are more durable, especially in women with a poor closure mechanism, since the forces created by a sling in a more horizontal direction are significantly lower than the forces created by a sling in a vertical direction [7]. This biomechanical concept is supported by clinical data showing that the retropubic approach is better 
in patients with low maximum urethral closure pressure (MUCP) than the transobturator approach if the MUCP is $<30 \mathrm{~cm} \mathrm{H}_{2} \mathrm{O}$ [8].

To compare different techniques, more domains than "efficacy" should be taken into consideration [9]. Regarding MUS-associated complications, the quality of studies focusing on this aspect is currently poor [10]. Consequently, the true incidence of MUS-related complications is unknown. However, the overall risk of a negative outcome has been estimated to be $\geq 15 \%$ [10]. The profile of morbidity after MUS seems to be different and a slightly higher after the retropubic procedure than after the transobturator procedure. Groin pain seems to be an issue after the transobturator procedure while voiding dysfunction seems to be a slightly higher after the retropubic procedure. Regarding the "economy" domain, there have been a few studies suggesting that the transobturator procedure may be more cost-effective than the retropubic procedure. However, taking into account the reoperation rate, it is possible that the retropubic procedure is more cost-effective.

In conclusion, accumulating medium-term and long-term data on reoperation rates, especially from two large national cohorts, indicate that MUS placed using the retropubic approach are significantly more durable than those placed using the transobturator approach. Therefore, it is time to question the commonly held view that the two techniques are equally effective. Since there are only minor differences in complication profiles and other domains of comparison, the retropubic procedure should as a rule of thumb be considered the primary choice. Obviously, there may be situations where the transobturator procedure is the preferred choice, e.g. in patients with voiding dysfunction, in patients with an abdominal hernia mesh, and in patients with multiple intraabdominal surgery. Surgeons should know about the significant difference in reoperation rates and the patient should be informed so that proper informed consent can be obtained before surgery. Preoperative measurement of MUCP may be of value in the preoperative work-up if both procedures are under consideration.

There are some important lessons to learn from this "MUS story" so far, namely that even after 20 years we still have many unresolved issues including uncertainties concerning MUS durability and safety when comparing the retropubic and transobturator procedures. Furthermore, randomized controlled trials and meta-analysis cannot stand alone in the assessment of surgical procedures especially regarding the issue of durability; national registries are an important source of supplementary data.

\section{References}

1. Ulmsten U, Henriksson L, Johnson P, Varhos G. An ambulatory surgical procedure under local anesthesia for treatment of female urinary incontinence. Int Urogynecol J Pelvic Floor Dysfunct. 1996;7(2):81-5.

2. Delorme E. Transobturator urethral suspension: mini-invasive procedure in the treatment of stress urinary incontinence in women. Prog Urol. 2001;11(6):1306-13.

3. Ford AA, Rogerson L, Cody JD, Ogah J. Mid-urethral sling operations for stress urinary incontinence in women. Cochrane Database Syst Rev. 2015;7:CD006375.

4. Foss Hansen M, Lose G, Kesmodel US, Gradel KO. Reoperation for urinary incontinence: a nationwide cohort study, 1998-2007. Am J Obstet Gynecol. 2016;214(2):263.e1-e8.

5. The Scottish Government. Scottish independent review of the use, safety and efficacy of transvaginal mesh implants in the treatment of stress urinary incontinence and pelvic organ prolapse in women. Edinburgh: The Scottish Government; 2017. http://www.gov.scot/Resource/0051/00515856.pdf. Accessed 23 Jun 2017.

6. Ford AA, Ogah JA. Retropubic or transobturator mid-urethral slings for intrinsic sphincter deficiency-related stress urinary incontinence in women: a systematic review and meta-analysis. Int Urogynecol J. 2016;27(1):19-28.

7. Schäfer W. Some biomechanical aspects of continence function. Scand J Urol Nephrol Suppl. 2001;207:44-60.

8. Tomoe H. Value of maximum urethral closure pressure in predicting the outcome of tension-free vaginal tape and transobturator tape procedure. Low Urin Tract Symptoms. 2013;5(2):65-8.

9. Lose G, Ostergard DR. Medical technology assessment and surgery for stress incontinence. Int Urogynecol J Pelvic Floor Dysfunct. 1999;10(6):351-2.

10. Blaivas JG, Purohit RS, Benedon MS, et al. Safety considerations for synthetic sling surgery. Nat Rev Urol. 2015;12(9):481-509. 